\title{
Update on Apraxia
}

\author{
Rachel Goldmann Gross, MD and Murray Grossman, MD \\ Department of Neurology, 2 Gibson, Hospital of the University of Pennsylvania, 3400 Spruce Street, \\ Philadelphia, PA 19104, USA. E-mail:mgrossma@mail.med.upenn.edu
}

\begin{abstract}
Apraxia is classically defined as difficulty performing learned, skilled gestures. In this review, we describe the range of motor impairments classified as apraxia, focusing on ideomotor limb apraxia. We present several prominent models of praxis to explain the variety of difficulties seen in patients with apraxia. We also discuss the large-scale frontal-parietal-basal ganglia network thought to underlie praxis. In this context, we highlight the common occurrence of limb apraxia in corticobasal degeneration, a neurodegenerative condition characterized by frontal, parietal, and basal ganglia disease.
\end{abstract}

\section{Introduction}

Apraxia has been defined as the inability to carry out learned, skilled motor acts despite preserved motor and sensory systems, coordination, comprehension, and cooperation [1]. This review focuses on ideomotor apraxia, and we briefly discuss other disorders of action that have been called apraxia as well as the controversy surrounding some of these designations. Moreover, we review the neuroanatomic localization of praxis and some influential models that have contributed to our understanding of the cognitive neuroscience of action.

It is widely agreed that the praxis system is made up of component functions associated with particular brain regions. These brain regions work together to process action. The network of structures underlying praxis is thought to include the frontal and parietal cortex, basal ganglia, and white matter tracts containing projections between these areas [2]. In this review, we highlight apraxia in corticobasal degeneration (CBD), a neurodegenerative condition classically described as an asymmetric akinetic-rigid syndrome with cortical features including apraxia [3]. This disorder is characterized by frontal, parietal, and basal ganglia dysfunction [4•]. CBD thus provides an informative model through which to explore the neuroanatomic correlates of praxis.

\section{Major Types of Apraxia}

\section{Ideomotor apraxia}

The most widely recognized type of apraxia is ideomotor apraxia, or impaired performance of skilled motor acts despite intact sensory, motor, and language function [1]. Ideomotor apraxia is typically demonstrated when a patient is asked verbally to perform a gesture with a limb. Most authors also include the inability to imitate another person's gesture, to perform the appropriate action in response to a visually presented object, or to carry out a movement using the actual object. Although controversial, many authors also consider difficulty with meaningless gestures (invented hand postures) as apraxia. Patients with ideomotor apraxia 
show temporal and spatial errors affecting timing, sequencing, amplitude, configuration, and limb position in space. They frequently use their limb as an object rather than demonstrating how to use the object [5-9]. Patients are often able to perform the same acts without difficulty in their daily lives. This phenomenon has been called the "voluntary-automatic dissociation" $[2,6,7,10]$. However, recent work in patients with left hemisphere stroke has shown that severity of ideomotor apraxia is associated with dependence on caregivers for certain physical activities of daily living [11].

Patients with ideomotor apraxia may exhibit differing degrees of impairment depending on testing conditions. For instance, patients typically have greatest difficulty performing gestures elicited by verbal command. They tend to have less difficulty imitating a gesture or acting in response to a visually presented object. They may be least impaired when asked to use the object itself [7,12-17]. Nevertheless, there are many reports that contradict these typical patterns $[14,17,18]$. Performance may differ depending on gesture type: transitive (involving an object; eg, using a hammer) versus intransitive (eg, waving goodbye); meaningful versus meaningless. Given these dissociations, it is essential that praxis assessments include a wide variety of tasks [6]. Indeed, task differences likely contribute to inconsistent findings across studies and patient populations.

\section{Ideational and conceptual apraxia}

Most authors would distinguish another type of apraxia-ideational apraxia—from ideomotor apraxia. In this condition, patients have difficulty carrying out a sequence of actions in performance of a complex, multistep task (eg, making a cup of tea). Ideational apraxia is often seen in patients with extensive left hemisphere damage, dementia, or delirium $[1,19]$. Thus, difficulty sequencing actions may not represent a higher-order motor programming deficit per se; rather, this deficit may be due to a combination of executive, language, and memory limitations [19] or to a general limitation in cognitive resources [20]. Some authors have made a distinction between ideational and conceptual apraxia $[1,21]$. Patients with conceptual apraxia show impairment of object or action knowledge. They may misuse objects, have difficulty matching objects and actions, be unaware of the mechanical advantage afforded by tools, or be unable to judge whether a gesture is well- or ill-formed [1,2,6,21]. Conceptual deficits can be seen in patients with dementia and have been associated with lesions of posterior regions of the left hemisphere $[1,21-24]$. Patients with ideational and conceptual apraxia tend to be profoundly disabled by their deficits in everyday life [2].

\section{Orofacial apraxia}

Orofacial apraxia is characterized by an impairment of skilled movements involving the face, mouth, tongue, larynx, and pharynx (eg, blowing a kiss). Orofacial apraxia has been associated with inferior frontal, deep frontal white matter, insula, and basal ganglia lesions [25]. Analogously to ideomotor limb apraxia, automatic movements of the same muscles are often preserved [25]. Moreover, orofacial apraxia frequently coexists with limb apraxia. Based on these observations, orofacial apraxia has been considered a subtype of ideomotor apraxia. However, orofacial and limb apraxia can be dissociated, suggesting that the neural systems underlying these disorders are at least partially separable [25].

\section{Limb-kinetic apraxia}

The term limb-kinetic apraxia has been used to describe inaccurate or clumsy distal limb movements. Limb-kinetic apraxia is often seen in the limb contralateral to the affected hemisphere, regardless of side [1,2]. However, Heilman and colleagues [26] used selective hemisphere anesthesia to demonstrate left hemisphere dominance for motor deftness in righthanded epilepsy patients with typical, left-sided language lateralization. The nature of limbkinetic apraxia has been controversial. This disorder has been associated with frontal lesions 
and can be difficult to differentiate from concurrent limb weakness [2]. Limb-kinetic apraxia has also been observed in patients with neurodegenerative conditions such as CBD [16], progressive supranuclear palsy [27], and Parkinson's disease [28], although it can be challenging to differentiate limb-kinetic apraxia from the extrapyramidal features of these disorders [15]. Limb-kinetic apraxia differs from classical ideomotor apraxia in a number of ways. For instance, limb-kinetic apraxia tends to be independent of modality (eg, verbal command versus imitation), and there is typically no voluntary-automatic dissociation [2].

\section{Other dyspraxias}

Several other types of motor dysfunction, including gait, speech, writing, constructional, and dressing apraxias, have been debated in the literature. In contrast to ideomotor apraxia, conditions such as speech and gait apraxia do not involve actions that must be explicitly learned. Moreover, these labels have been applied to a wide variety of clinical phenomena. For instance, the term gait apraxia has been used to describe the gait of normal pressure hydrocephalus, vascular parkinsonism, and several other disorders affecting the frontal lobes or frontostriatal connections [29]. These disorders may be associated with other neurologic deficits, including weakness, frontal dysfunction, aphasia, inattention, or neglect. For instance, constructional apraxia, or the inability to copy visually presented information, can be seen in patients with right parietal lesions, where it is likely due to a higher-order visuospatial processing deficit, or in patients with frontal dysfunction, where there is impaired organization and planning [30].

Finally, it is important to consider the relationship between limb apraxia and aphasia. In patients with left hemisphere damage, apraxia and aphasia frequently coexist. This observation begs the question of whether linguistic dysfunction is responsible for apraxia [13]. It is possible that in some cases aphasia contributes to patients' greater difficulty with gestures elicited by verbal command relative to those elicited in other ways. However, aphasia cannot account for apraxia in all circumstances (eg, gesture imitation). Moreover, dissociations between apraxia and aphasia have been reported [6]. Thus, rather than a causal relationship between aphasia and apraxia, these two functions may be frequently affected in the same patient because of a single lesion affecting adjacent neural substrates for language and gesture. More detailed discussions of the relation between apraxia and aphasia are presented by Petreska et al. [6] and Pramstaller and Marsden [31].

\section{Anatomy of Ideomotor Limb Apraxia}

Praxis has long been considered a left hemisphere function. For instance, apraxia occurs more frequently in patients with left versus right hemisphere injury [7,13,31,32]. Left hemisphere dominance also has also been supported by functional imaging studies showing activation of the left hemisphere when gestures are performed with either hand [33]. In addition, left hemisphere damage tends to cause a more severe and extensive apraxia $[7,13]$. Nevertheless, apraxia has been seen after focal injury or neurodegeneration affecting the right hemisphere, suggesting that right-sided structures can also support skilled movements [7,13,34,35•]. This may explain sparing of some aspects of praxis (eg, intransitive gestures, gesture recognition) after left hemisphere injury [10]. Moreover, an electroencephalogram (EEG) coherence analysis in patients with apraxia after left hemisphere stroke who practiced a set of tool-use pantomimes revealed a more robust right frontoparietal network relative to controls, suggesting that the right hemisphere can be recruited in the face of injury or as a result of training [35•].

The relative involvement of the hemispheres in praxis may depend on the type of motor task and the context in which it is performed. The left hemisphere has been implicated in processing movements of relatively high complexity, planning and sequencing movements, shifting motor attention, and selecting a motor response from a set of competitors [7,36-38]. In addition, investigators have suggested that patients with left hemisphere injury are more impaired in less 
naturalistic testing conditions (eg, performing gestures to verbal command versus use of an actual object) and that the left hemisphere is specialized for carrying out motor acts in the abstract or in a context-independent manner [7]. This view is consistent with the observation that some patients with left hemispheric injury who demonstrate apraxia in the clinic or laboratory have relatively less impairment in their daily lives $[7,10]$. The right hemisphere, in contrast, is called to action for highly practiced, familiar actions [10].

In addition to establishing hemispheric dominance, attempts have been made to localize praxis within the left hemisphere. From its earliest conceptions, ideomotor apraxia has been associated with damage to parietal and frontal cortex as well as the white matter connections between these areas. These structural-functional correlations are largely based on patients with focal lesions (eg, stroke) or with neurodegenerative conditions affecting frontoparietal areas (eg, CBD) $[1,15,23,39 \bullet, 40]$. In addition to lesion studies, imaging has helped establish the neural correlates of praxis. Using MRI and CT in patients with left hemisphere strokes, Haaland et al. [32] identified areas around the left intraparietal sulcus (Brodmann areas 7, 39, and 40) and left middle frontal gyrus (Brodmann areas 9, 46, 6, 8) as regions associated with apraxia. Moll et al. [33] reported that functional MRI during gesture performance in neurologically intact individuals revealed activation in left frontal and parietal regions, including the dorsolateral frontal and intraparietal cortex [33]. Kareken et al. [41] performed a positron emission tomography (PET) study of a 65-year-old woman with progressive neurologic deficits including apraxia and showed reduced uptake in left frontal (supplementary motor area) and parietal (angular gyrus, supramarginal gyrus) regions [41].

Ideomotor apraxia may be seen following injury to brain regions other than the frontal and parietal areas typically associated with apraxia. For example, apraxia can result from anterior callosal lesions. Apraxia in this setting tends to be unilateral involving the left hand. The lesion presumably disconnects right hemisphere premotor and motor cortex from left hemisphere praxis input, underscoring left hemisphere dominance in the control of praxis $[2,6,40]$.

The basal ganglia also play an important role in praxis, likely via connections with frontal and parietal cortex [5,29]. Ideomotor apraxia has been demonstrated in patients with subcortical vascular injury [7,12]. Apraxia also has been seen in patients with various movement disorders, including CBD [4•,8,9,15,42], Parkinson's disease [27], progressive supranuclear palsy [27, 42], and Huntington's disease [43], all of which compromise the basal ganglia. Proposed contributions of the basal ganglia to praxis include sequencing, fine tuning of movements, selection of competing motor programs, and performance of automatic or overlearned movements [5]. Nevertheless, it is not clear that isolated basal ganglia dysfunction causes significant apraxia. Most cases also involve surrounding white matter tracts and frontoparietal cortex $[27,31]$.

As with most complex behaviors, it is likely that praxis depends on a complex, large-scale network of structures [2,29]. Imaging studies involving connectivity analysis are essential in defining this network. In one such study, Wheaton et al. [44••] performed an EEG coherence analysis of praxis in neurologically intact individuals. This study revealed two coherent circuits: one involving parietal cortex, the supplementary motor area, and the motor cortex and the other involving parietal, lateral premotor, and motor areas [44••]. Finding a network composed of multiple, partially separable sets of structures could explain discrepancies across studies regarding the particular structures within frontal or parietal cortex associated with apraxia [7,12]. Indeed, various tasks may differentially engage portions of this network, and various diseases may differentially compromise portions of this network $[6,15]$. 


\section{Neuropsychological Models of Apraxia}

Numerous authors have contributed to the understanding of the neuropsychological basis of apraxia. We review some of the most influential theories here. The reader is referred to the individual citations for more in-depth description of each model and how each predicts performance under various testing conditions.

Liepmann [23] proposed that the representation of an action (space-time plan) is stored in the left parietal lobe. To execute the action, the space-time plan is retrieved and, via the left premotor cortex, information is conveyed to primary motor areas. For the left arm to perform an action, information crosses via the corpus callosum to right premotor areas. In patients with ideomotor apraxia, motor representations and limb kinetics are intact; the inability to act is due to disruption of frontoparietal connections. Ideational apraxia was thought to result from disruption of action representations in the left parietal lobe and limb-kinetic apraxia from disruption of "kinaesthetic-innervatory engrams" in the left frontal lobe [23].

In his model of apraxia, Geschwind [40] conceived of a disconnection between motor and more posterior language areas. The disruption was thought to involve the superior longitudinal fasciculus that connects Wernicke's area to left frontal cortex. A lesion in this pathway would spare comprehension but compromise performance of actions elicited by verbal command [40]. To account for impaired gesture imitation (which does not require intact language function) in patients with apraxia, Geschwind [45] proposed that visual association areas and premotor cortex are connected via the same white matter tracts running between language and motor areas, but this notion remains controversial [1].

Subsequent authors have provided evidence supporting dual-component models of praxis $[22,24,38,46]$. In one of the most influential models, Rothi and Heilman and colleagues [22, 24] propose anterior execution-production and posterior conceptual-representational components. Knowledge and representations of objects and actions are thought to be stored in the left parietal lobe (including the angular gyrus and supramarginal gyrus) and then transformed into a signal by the premotor cortex (including the supplementary motor area), which is then used by motor cortex to carry out the action [24]. Damage to anterior regions causes a gesture production deficit. Patients with posterior apraxia typically have impaired movement production as well as difficulty with gesture comprehension and discrimination of well- versus ill-formed gestures [24]. Support for this anterior-posterior dissociation has come from lesion studies showing impaired gesture recognition after posterior, but not anterior, left hemispheric injury [22]. Imaging studies have less consistently shown such a relationship $[15,41,47]$.

Buxbaum and colleagues $[39 \bullet, 46]$ proposed a model of praxis that involves the left inferior parietal lobe and a frontoparietal information processing system. According to this model, the inferior parietal lobe processes internal representations of movements and body part position. The frontoparietal processing system is responsible for spatial-motor transformation. Damage to this component results in "dynamic apraxia" due to disruption of the process by which gesture representations, having incorporated information about current and intended body part positions, are transformed into motor programs for action.

Impaired imitation of meaningless gestures is often included in descriptions of patients with apraxia. However, it is not clear that this deficit represents apraxia as classically defined. Indeed, meaningless gestures are invented postures, not learned skilled actions. It may be that this deficit has a different basis (eg, impaired visuospatial mediation of body part relationships) but frequently coexists with ideomotor apraxia because of a partially shared anatomic substrate [39•]. Nevertheless, the study of meaningless gestures has helped refine models of praxis. For instance, dissociations in performance of meaningful versus meaningless gestures has 
suggested that, in addition to a route requiring access to stored concepts and representations, there is a more direct pathway for processing invented hand postures that bypasses this step [24,39•,48]. This notion of separable processing routes is supported by Moll et al. [33], who demonstrated functional MRI activation in a particular part of the parietal lobe during processing of tool-use gestures but not meaningless gestures.

Recently, interest has grown in areas of the parietal lobe used in higher-order information processing and integration. For instance, some authors have proposed that object use involves information processed by the ventral (occipitotemporal) and dorsal (parietooccipital) streams and that these two types of information are integrated by the inferior parietal lobe [39•]. Moreover, Goldenberg and Hagmann [48] provide evidence for impaired manipulation or elaboration of gesture representations in patients with left angular gyrus lesions. Work in our own laboratory has shown evidence of impaired conceptual integration in patients with CBD, who are known to have prominent parietal lobe dysfunction [49].

The discovery of mirror neurons represents a great advance in the understanding of how the brain processes action. Indeed, particular neuronal populations have been identified that are activated when an action is produced and when an individual observes that same action $[50 \bullet \bullet, 51]$. These findings may pose a challenge for models stipulating separate structures specialized for action execution versus action recognition or comprehension [6]. A new model would have to account for the dissociations between gesture production and reception seen in individuals with a variety of underlying neuropathologies. The mirror neuron system thus may have a substantial impact on how we understand the relation between expressive and receptive praxis and how these are integrated into a larger praxis network.

\section{Apraxia in Corticobasal Degeneration}

CBD is a neurodegenerative condition classically described as an asymmetric akinetic-rigid syndrome with cortical features such as apraxia as well as the alien-limb phenomenon and cortical sensory loss [3]. Ideomotor apraxia is frequently seen in patients with CBD. In a recent series of 15 patients with autopsy-proven CBD, Murray et al. [4•] showed that $40 \%$ of patients had apraxia at disease onset, and $72 \%$ had apraxia at the time of death. Patients had predominantly ideomotor limb apraxia, and fewer exhibited orofacial apraxia. CBD is characterized by atrophy $[4 \cdot 52 \bullet \bullet]$ and decreased metabolism [17] in the network of frontal, parietal, and basal ganglia structures thought to underlie praxis.

Graham et al. [15] attempted to characterize apraxia in a patient with CBD. The patient had impaired gesture production and relatively preserved receptive praxis, suggesting an anterior apraxia. The patient's performance improved significantly when he manipulated an actual object or saw the target of an action. As argued for patients with left hemisphere vascular injury [7], it may be that more naturalistic settings (ie, holding an object or seeing the target of an action) facilitated gesture performance in this patient with left hemisphere-predominant CBD. Indeed, at the time of testing, the patient had relatively preserved activities of daily living. Of note, the patient had an alien limb and severe utilization behavior, suggesting that patients with CBD may exhibit extreme (and deleterious) context dependence [15]. Performance may have been bolstered by visual or tactile cues that provide semantic information or constrain the set of possible limb configurations or movements [15].

It is also important to consider the role of visual cues in patients with CBD who frequently have cortical sensory loss. A lack of sensory feedback in CBD could impede motor programming and execution, and this could be mitigated by providing visual information [9]. Nevertheless, it is not clear that cortical sensory loss contributes to apraxia in all patients with CBD. Indeed, patients have been described who do not benefit from visual input $[9,15]$. Moreover, not all patients with apraxia have cortical sensory loss [8]. 
Several authors have attempted to characterize apraxia in CBD within the framework of dualcomponent models of praxis [24]. For instance, Jacobs et al. [9] studied four patients with early CBD and no significant cognitive impairment. Consistent with anterior apraxia, the patients had difficulty with gesture production, relative sparing of gesture recognition, and impaired imitation of meaningful and nonsense gestures, both of which depend on an intact production system [9]. Leiguarda et al. [8] demonstrated ideomotor apraxia in seven of 10 patients with CBD. There was a correlation between ideomotor apraxia and performance on a picture arrangement task. Because this task requires planning and abstraction, the authors attributed ideomotor apraxia in their patients to frontal dysfunction. However, three patients also had ideational apraxia (difficulty with complex, multistep tasks) and impaired receptive praxis (gesture comprehension and recognition), two of whom had severe cognitive impairment. Thus, the authors hypothesized that additional involvement of the parietal lobe, or more diffuse cortical degeneration, caused a conceptual praxis impairment over time in this subgroup of patients [8].

Imaging studies have attempted to establish neural correlates of apraxia in patients with CBD. Peigneux et al. [17] used PET to compare metabolism in patients with and without apraxia. Apraxia was defined using two different measures: a performance score (percentage of correct gestures on an initial trial) and a correction score (proportion of errors corrected on a second attempt). Patients with apraxia as defined by the performance score showed decreased metabolism in the anterior cingulate cortex. Using the correction score, the authors demonstrated hypometabolism in contralateral frontal and parietal regions, including the supplementary motor area and superior parietal lobule, respectively.

Borroni et al. [52••] studied 20 patients with CBD using MRI voxel-based morphometry and diffusion tensor imaging. The volumetric analysis showed atrophy in frontal and parietal regions as well as the corpus callosum. Diffusion tensor imaging revealed structural abnormality of association fibers between the frontal and parietal lobes, within the parietal lobe, within hand cortical areas, and across the corpus callosum. Gesture imitation correlated with bilateral parietal volumes and with structural abnormality of left frontoparietal association fibers. Moreover, limb-kinetic apraxia was correlated with structural abnormality of hand sensorimotor connecting fibers.

\section{Conclusions}

This article has reviewed the major types of apraxia with a focus on the clinical features, neuroanatomic correlates, and cognitive neuroscience of ideomotor apraxia. Lesion and imaging studies have revealed a network of structures underlying praxis, including frontal and parietal cortex, the basal ganglia, and the white matter tracts between them. We highlighted apraxia in CBD, a neurodegenerative condition that involves this network of structures. The studies we have described vary in the relative contribution they reported of frontal versus parietal dysfunction to apraxia in patients with CBD. Clinically, these patients vary in the relative degree of frontal versus parietal dysfunction they exhibit. Ultimately, to appreciate the full spectrum of praxis impairment in CBD and how various brain regions contribute to apraxia in these patients will require studies with larger sample sizes that use functional imaging techniques during task performance [29].

Attempts to understand the nature of apraxia are complicated by several issues. First, there is an extremely wide range of possible actions to be studied. A particular gesture may be associated with different processing demands depending on how it is elicited [6]. Moreover, not all authors agree on what types of motor impairment should be considered apraxia. Finally, studies use different patient populations, lesion sizes, praxis tasks, criteria for defining apraxia, means of error assessment, and imaging techniques. 
The study of praxis in health and disease nevertheless has important implications for fundamental neuroscientific questions, including localization of function, hemispheric potential for praxis, and the ability of the brain to compensate for injury. A goal for research in this area is more detailed mapping of large-scale networks underlying praxis, with serial assessment of alterations in these networks following injury or during learning. Greater understanding of these issues could help guide the development of innovative therapeutic and rehabilitative interventions.

\section{Acknowledgments}

Disclosures

This work was supported in part by National Institutes of Health grants NS44266, NS53488, AG17586, and AG15116.

\section{References and Recommended Reading}

Papers of particular interest, published recently, have been highlighted as:

- Of importance

•• Of major importance

1. Heilman, KM.; Rothi, LJG. Apraxia. In: Heilman, KM.; Valenstein, E., editors. Clinical Neuropsychology. New York: Oxford University Press; 2003. p. 215-235.

2. Leiguarda RC, Marsden CD. Limb apraxias: higher-order disorders of sensorimotor integration. Brain 2000;123(Pt 5):860-879. [PubMed: 10775533]

3. Mahapatra RK, Edwards MJ, Schott JM, Bhatia KP. Corticobasal degeneration. Lancet Neurol 2004;3:736-743. [PubMed: 15556806]

4. Murray R, Neumann M, Forman MS, et al. Cognitive and motor assessment in autopsy-proven corticobasal degeneration. Neurology 2007;68:1274-1283.1283 [PubMed: 17438218] This article describes the cognitive and motor features of CBD. Its major strengths include use of autopsy-proven cases and a fine-grained analysis of cognitive function. The authors show that CBD patients have a specific profile of cognitive impairment related to the neuroanatomic distribution of disease.

5. Leiguarda R. Limb apraxia: cortical or subcortical. Neuroimage 2001;14:S137-S141. [PubMed: 11373145]

6. Petreska B, Adriani M, Blanke O, Billard AG. Apraxia: a review. Prog Brain Res 2007;164:61-83. [PubMed: 17920426]

7. Schnider A, Hanlon RE, Alexander DN, Benson DF. Ideomotor apraxia: behavioral dimensions and neuroanatomical basis. Brain Lang 1997;58:125-136. [PubMed: 9184099]

8. Leiguarda R, Lees AJ, Merello M, et al. The nature of apraxia in corticobasal degeneration. J Neurol Neurosurg Psychiatry 1994;57:455-459. [PubMed: 8163995]

9. Jacobs DH, Adair JC, Macauley B, et al. Apraxia in corticobasal degeneration. Brain Cogn 1999;40:336-354. [PubMed: 10413565]

10. Rapcsak SZ, Ochipa C, Beeson PM, Rubens AB. Praxis and the right hemisphere. Brain Cogn 1993;23:181-202. [PubMed: 8292325]

11. Hanna-Pladdy B, Heilman KM, Foundas AL. Ecological implications of ideomotor apraxia: evidence from physical activities of daily living. Neurology 2003;60:487-490. [PubMed: 12578932]

12. Hanna-Pladdy B, Heilman KM, Foundas AL. Cortical and subcortical contributions to ideomotor apraxia: analysis of task demands and error types. Brain 2001;124:2513-2527. [PubMed: 11701604]

13. De Renzi E, Motti F, Nichelli P. Imitating gestures. A quantitative approach to ideomotor apraxia. Arch Neurol 1980;37:6-10. [PubMed: 7350907]

14. De Renzi E, Faglioni P, Sorgato P. Modality-specific and supramodal mechanisms of apraxia. Brain 1982;105:301-312. [PubMed: 6177376] 
15. Graham NL, Zeman A, Young AW, et al. Dyspraxia in a patient with corticobasal degeneration: the role of visual and tactile inputs to action. J Neurol Neurosurg Psychiatry 1999;67:334-344. [PubMed: 10449556]

16. Leiguarda RC, Merello M, Nouzeilles MI, et al. Limb-kinetic apraxia in corticobasal degeneration: clinical and kinematic features. Mov Disord 2003;18:49-59. [PubMed: 12518300]

17. Peigneux P, Salmon E, Garraux G, et al. Neural and cognitive bases of upper limb apraxia in corticobasal degeneration. Neurology 2001;57:1259-1268. [PubMed: 11591846]

18. Merians AS, Clark M, Poizner H, et al. Apraxia differs in corticobasal degeneration and left-parietal stroke: a case study. Brain Cogn 1999;40:314-335. [PubMed: 10413564]

19. Weintraub, S. Neuropsychological assessment of mental state. In: Mesulam, MM., editor. Principles of Behavioral and Cognitive Neurology. New York: Oxford University Press; 2000. p. 135-136.

20. Giovannetti T, Libon DJ, Buxbaum LJ, Schwartz MF. Naturalistic action impairments in dementia. Neuropsychologia 2002;40:1220-1232. [PubMed: 11931925]

21. Ochipa C, Rothi LJ, Heilman KM. Conceptual apraxia in Alzheimer's disease. Brain 1992;115(Pt 4): 1061-1071. [PubMed: 1393502]

22. Heilman KM, Rothi LJ, Valenstein E. Two forms of ideomotor apraxia. Neurology 1982;32:342346. [PubMed: 7199656]

23. Liepmann H. Apraxie. Ergebn ges Med 1920;1:516-543.

24. Rothi, LGJ.; Ochipa, C.; Heilman, KM. A cognitive neuropsychological model of limb praxis and apraxia. In: Rothi, LJG.; Heilman, KM., editors. Apraxia: The Neuropsychology of Action. East Sussex, UK: Psychological Press; 1997. p. 28-49.

25. Ozsancak C, Auzou P, Dujardin K, et al. Orofacial apraxia in corticobasal degeneration, progressive supranuclear palsy, multiple system atrophy and Parkinson's disease. J Neurol 2004;251:1317-1323. [PubMed: 15592726]

26. Heilman KM, Meador KJ, Loring DW. Hemispheric asymmetries of limb-kinetic apraxia: a loss of deftness. Neurology 2000;55:523-526. [PubMed: 10953184]

27. Leiguarda RC, Pramstaller PP, Merello M, et al. Apraxia in Parkinson's disease, progressive supranuclear palsy, multiple system atrophy and neuroleptic-induced parkinsonism. Brain 1997;120 (Pt 1):75-90. [PubMed: 9055799]

28. Quencer K, Okun MS, Crucian G, et al. Limb-kinetic apraxia in Parkinson disease. Neurology 2007;68:150-151. [PubMed: 17151340]

29. Zadikoff C, Lang AE. Apraxia in movement disorders. Brain 2005;128:1480-1497. [PubMed: 15930045]

30. Damasio, AR.; Tranel, D.; Rizzo, M. Disorders of complex visual processing. In: Mesulam, MM., editor. Principles of Behavioral and Cognitive Neurology. New York: Oxford University Press; 2000. p. 362-363.

31. Pramstaller PP, Marsden CD. The basal ganglia and apraxia. Brain 1996;119(Pt 1):319-340. [PubMed: 8624692]

32. Haaland KY, Harrington DL, Knight RT. Neural representations of skilled movement. Brain 2000;123 (Pt 11):2306-2313. [PubMed: 11050030]

33. Moll J, de Oliveira-Souza R, Passman LJ, et al. Functional MRI correlates of real and imagined tooluse pantomimes. Neurology 2000;54:1331-1336. [PubMed: 10746606]

34. Hanna-Pladdy B, Daniels SK, Fieselman MA, et al. Praxis lateralization: errors in right and left hemisphere stroke. Cortex 2001;37:219-230. [PubMed: 11394722]

35. Wheaton LA, Bohlhalter S, Nolte G, et al. Cortico-cortical networks in patients with ideomotor apraxia as revealed by EEG coherence analysis. Neurosci Lett 2008;433:87-92.92 [PubMed: 18249498] This study used EEG coherence, a type of connectivity analysis that establishes a network of structures defined by temporally related areas of cortical activity. In patients with apraxia due to stroke or CBD, the authors showed involvement of right hemisphere structures in a praxis network. This finding has important implications for right hemisphere capacity for praxis and ability to mediate recovery.

36. Rushworth MF, Nixon PD, Renowden S, et al. The left parietal cortex and motor attention. Neuropsychologia 1997;35:1261-1273. [PubMed: 9364496] 
37. Rushworth MF, Nixon PD, Wade DT, et al. The left hemisphere and the selection of learned actions. Neuropsychologia 1998;36:11-24. [PubMed: 9533383]

38. Roy, EA.; Square, PA. Neuropsychology of movement sequencing disorders and apraxia. In: Zeidel, DW., editor. Neuropsychology. London: Academic Press; 1994. p. 183-218.

39. Buxbaum LJ, Kyle K, Grossman M, Coslett HB. Left inferior parietal representations for skilled handobject interactions: evidence from stroke and corticobasal degeneration. Cortex 2007;43:411423.423 [PubMed: 17533764] The authors provide a model of praxis that emphasizes the role of body knowledge and how it is incorporated in a dynamic way as motor programs are translated into action. Their model predicts different patterns of praxis behavior in patients with CBD versus those with left parietal stroke. They also discuss the inferior parietal lobe as an integrator of object knowledge and body representations.

40. Geschwind N. Disconnexion syndromes in animals and man. Brain 1965;88:237-294. 585-644. [PubMed: 5318481]

41. Kareken DA, Unverzagt F, Caldemeyer K, et al. Functional brain imaging in apraxia. Arch Neurol 1998;55:107-113. [PubMed: 9443717]

42. Soliveri P, Piacentini S, Girotti F. Limb apraxia in corticobasal degeneration and progressive supranuclear palsy. Neurology 2005;64:448-453. [PubMed: 15699373]

43. Hamilton JM, Haaland KY, Adair JC, Brandt J. Ideomotor limb apraxia in Huntington's disease: implications for corticostriate involvement. Neuropsychologia 2003;41:614-621. [PubMed: 12559154]

44. Wheaton LA, Nolte G, Bohlhalter S, et al. Synchronization of parietal and premotor areas during preparation and execution of praxis hand movements. Clin Neurophysiol 2005;116:1382-1390.1390 [PubMed: 15978500] Using EEG coherence, the authors demonstrate functional connectivity between the frontal and parietal cortex during task performance. This type of study is vital for identifying neural networks underlying praxis.

45. Geschwind N. The apraxias: neural mechanisms of disorders of learned movement. Am Sci 1975;63:188-195. [PubMed: 1115438]

46. Buxbaum LJ. Ideomotor apraxia: a call to action. Neurocase 2001;7:445-458. [PubMed: 11788737]

47. Peigneux P, Van der Linden M, Garraux G, et al. Imaging a cognitive model of apraxia: the neural substrate of gesture-specific cognitive processes. Hum Brain Mapp 2004;21:119-142. [PubMed: 14755833]

48. Goldenberg G, Hagmann S. The meaning of meaningless gestures: a study of visuo-imitative apraxia. Neuropsychologia 1997;35:333-341. [PubMed: 9051681]

49. Goldmann Gross, R.; Ash, S.; Anderson, C.; Grossman, M. Discourse impairment in corticobasal degeneration; Presented at the 2007 annual meeting of the American Neurological Association conference; Washington, DC: 2007 Oct.

50. Iacoboni M, Dapretto M. The mirror neuron system and the consequences of its dysfunction. Nat Rev Neurosci 2006;7:942-951.951 [PubMed: 17115076] The mirror neuron system provides an empirically based account for the intimate connection between frontal and parietal regions. This review catalogues critical functions of the frontoparietal system. For example, interruption of the mirror neuron system may result in apraxia by interfering with the intention to grasp.

51. Rizzolatti G, Craighero L. The mirror-neuron system. Annu Review Neurosci 2004;27:169-192.

52. Borroni B, Garibotto V, Agosti C, et al. White matter changes in corticobasal degeneration syndrome and correlation with limb apraxia. Arch Neurol 2008;65:796-801.801 [PubMed: 18541800] This study imaged both gray and white matter to characterize structural abnormalities in patients with CBD. The authors showed cortical atrophy in frontoparietal areas and disruption of association fibers within and between these regions, which they correlated with apraxia. These findings again emphasize that the neural correlate of praxis is best understood as a network of structures. 